\title{
Research on the System Symbiotic Evolution Equation of Maritime Transportation Economic Belt with Specific Scale Variables
}

\author{
Wen Wang* 1 \\ ${ }^{1}$ Navigation College Jimei University Xiamen, China
}

\begin{abstract}
In order to study the relationship between marine transportation and the development of coastal areas and their evolutionary trends, the part of maritime transportation is expanded in the theory of transportation economic belt. This paper takes the shipping volume of the shipping passage and the landward depth of the economic belt as the symbiotic scale variables, and establishes the unilateral non independent symbiosis evolution logistic equation of maritime transport economic belt. A quantitative approach is proposed to get the form of the interaction coefficients of the variables with theories of transportation economy, plume model, and calculus etc., solving the unknown coefficients, then obtains the increasing and decreasing factors of the interaction coefficients. Results show that the interaction coefficients of both scale variables decrease with the increase of their values.
\end{abstract}

\section{INTRODUCTION}

Modern Marine economy is an important support for building a powerful marine country. Developing marine shipping industry and the layout of coastal land are important for coastal countries. Then how does maritime transportation and industrial economy function and develop? How does its spatial form evolve? Maritime transportation economic belt and its evolution rules and development models have become important research needs in many aspects such as economy, trade, diplomacy, industrial development and maritime security. At present, the general concept and basic theory of transportation economic belt have been established, and a relatively systematic theoretical framework has been formed, and there is analysis of typical cases, but mainly concentrated on land transportation trunk lines. Based on theories of transportation economic belt and symbiosis, the system measurement variables are selected in this paper for the co-development of the shipping channel and its economic belt, and quantitatively studies their interaction mechanism, to provide a reference for further study on the theory of marine transportation economic belt.

\section{LITERATURE REVIEW}

The main theoretical basis includes the growth pole theory in the 1950s put forward by F. Perroux (a French economist), the growth axis theory in the early 1960s put forward by Werner sambart (a German scholar), the research on urban transportation corridor in the early 1990s by Edward C. Sullivan (an American scholar), and the theory of point-axis spatial structure system put forward by $\mathrm{Mr}$. Lu Dadao (Institute of geography, Chinese Academy of Sciences) in 1984. Then in 1993, Mr. Zhang Wuguo (Institute of system engineering of Northern Jiaotong University of China) established the concept of "transportation economic belt", and Mr. Zhang Wenchang (2002) further systematized the basic theory of transportation economic belt ${ }^{[1]}$. Early research mainly focused on the changes and development of the industrial belt along railway.

In recent international literature, the impact of transport corridors on regional and land planning is relatively close to the research direction of this paper. For example, Tsekeris proposed a dynamic space panel model to study how changes in regional transportation conditions affect its own and surrounding areas' ability to trade abroad $^{[2]}$, Clott et al. considered that the centralized freight corridor has a more direct impact on the freight planning of the regional transportation network than the decentralized regional freight ${ }^{[3]}$. There are also studies on the evaluation of traffic corridors, for example, Wiegmans et al. Analyzed, modeled and evaluated the performance of the supply chain of long-distance intercontinental land and sea freight corridors ${ }^{[4]}$, Dong et al. Evaluated the performance of two trade corridors between the Far East and Europe -- the China Europe sea land express and the new Eurasian land bridge through questionnaires ${ }^{[5]}$.

\section{THE CONCEPT OF MARITIME TRANSPORTATION ECONOMIC BELT}

The definition of transportation economic belt in literature [1]: taking the traffic trunk or comprehensive

${ }^{*}$ Corresponding author: wwns@jmu.edu.cn 
transportation channel as the main development axis, relying on the large and medium-sized cities on the axis or within its attraction scope, a developed strip-shaped economic region, which is dominated by developed industries, especially the secondary and tertiary industries, and is an economically dense belt with traffic infrastructure as the axis.

Transport corridor, industries, economic centers or large and medium-sized cities are the basic elements. Transport corridor is the precondition for the formation of transportation economic belt, and actively promotes population and industrial agglomeration and diffusion. Industry is the main component of economic belt. Industrial structure and its evolution and upgrading, industrial agglomeration and evacuation will promote the development and evolution of economic belt and extend it outwards. Large and medium-sized cities are the basis of population and industry, and are the growth poles of transportation economic belt, growing into economic centers and sub-centers, which is one of the spatial signs of the transportation economic belt.

Based on the existing views and statements, the maritime transportation economic belt in this paper is a belt-like regional economic system, and its axis is the maritime channel composed of several ports and port cities in the coastal area, mainly relying on the attraction of closely related cities and towns, urban and industrial agglomerations in the land area. Among the basic elements, the port and the shipping companies operating between them constitute the shipping corridor, and coastal ports themselves are usually larger economic centers. Differences in size, status and function of ports within the economic belt may result in trunk and feeder lines of the maritime channel. Bulk goods are of large proportion in the belt, which are suitable for the characteristics of low unit cost and less time requirement in shipping mode.

\section{The Symbiotic Relationship Between ShIPPING PASSAge AND ITS ECONOMIC BELT AND SCALE VARIABLES}

Symbiosis is an ecological theory, which means that different species live together according to a certain physical relationship. Later, it refers to the two symbiotic units cooperate with each other in function to improve their adaptation to the environment, and form a relationship of mutual dependence, complementary advantages, close links, and common development. Transportation channel and its economic belt obviously have a symbiosis relationship similar to that of biological population. The survival and development of various industries within the economic belt need transportation channel to provide passenger and cargo transportation services, and the existence and development of the transportation channel also need the support of passenger and cargo flow of relevant industries. Therefore, they are interdependent and complementary in demand.

To quantitatively study their interaction and development relationship, it is necessary to select the scale variables that represent their properties and states and establish logistic equation. In this paper, two representative indexes of shipping passage and its economic belt are selected as the scale variables, namely, the transport volume of shipping passage and the landward depth of the economic belt. The economic belt is distributed along the coast, and its landward depth refers to the width extending inland from the coastline. Establish the symbiotic equation for the shipping passage and its economic belt:

$$
\left\{\begin{array}{l}
\frac{d L_{e}}{d t}=a_{e} L_{e}\left(-1-\frac{L_{e}}{K_{e}}+\sigma_{e} \frac{Q_{s}}{K_{s}}\right) \\
\frac{d Q_{s}}{d t}=a_{s} Q_{s}\left(1-\frac{Q_{s}}{K_{s}}+\sigma_{s} \frac{L_{e}}{K_{e}}\right)
\end{array}\right.
$$

$\mathrm{L}_{e}$ is the landward depth of the maritime transport economic belt, and $\mathrm{Q}_{\mathrm{s}}$ is the transport volume of the shipping passage. $a_{e}, a_{s}$ are the natural growth rates of $L_{e}$ and $\mathrm{Q}_{\mathrm{s}}$ respectively, and $\mathrm{K}_{\mathrm{e}}$ and $\mathrm{K}_{\mathrm{s}}$ are the maximum values of separate $\mathrm{L}_{\mathrm{e}}$ and $\mathrm{Q}_{\mathrm{s}}$ that environmental resources can bear respectively. $\sigma_{\mathrm{e}}$ and $\sigma_{\mathrm{s}}$ are the influence coefficients of $\mathrm{Q}_{\mathrm{s}}$ on $\mathrm{L}_{\mathrm{e}}$ and $\mathrm{L}_{\mathrm{e}}$ on $\mathrm{Q}_{\mathrm{s}}$ respectively. According to the meaning of the symbiosis equation, $\sigma_{\mathrm{e}}$ means the number of $L_{e}$ that one unit $Q_{s}$ can increase, $\sigma_{s}$ is similar. $t$ is time. According to the definition of transport economic belt, the maritime transport economic belt cannot exist without shipping passage, therefore the coefficient of $a_{e} L_{e}$ in $L_{e}$ 's growth equation is -1 . Then the forms of $\sigma_{\mathrm{e}}$ and $\sigma_{\mathrm{s}}$ are the problems to be solved.

\section{InTERACTION Coefficients OF the SCALE VARIABLES}

\subsection{The Effect Coefficient $\sigma_{e}$}

Both $\mathrm{L}_{\mathrm{e}}$ and $\mathrm{Q}_{\mathrm{s}}$ are continuous, and $\sigma_{\mathrm{e}}$ to be solved is $\mathrm{dL}_{\mathrm{e}} / \mathrm{dQ}_{\mathrm{s}}$, that is, the change rate of $\mathrm{L}_{\mathrm{e}}$ to $\mathrm{Q}_{\mathrm{s}}$. $\mathrm{P}_{1}$ and $\mathrm{P}_{2}$ are the points on the inland boundary of the economic belt, the distance to the respective ports $\mathrm{H}_{1}$ and $\mathrm{H}_{2}$ is $\mathrm{L}_{\mathrm{e}}$ and $\mathrm{L}_{\mathrm{e}}$ '. Distance between $\mathrm{H}_{1}$ and $\mathrm{H}_{2}$ is $\mathrm{L}$. Transportation between $\mathrm{P}_{1}$ and $\mathrm{P}_{2}$ can also be land transportation with a distance of $\mathrm{L}_{0}$. The unit transportation costs of $\mathrm{P}_{1}-\mathrm{H}_{1}$ land transportation, $\mathrm{H}_{2}-\mathrm{P}_{2}$ land transportation, $\mathrm{H}_{1}-\mathrm{H}_{2}$ sea transportation and $\mathrm{P}_{1}-\mathrm{P}_{2}$ land transportation are $\mathrm{c}_{1}, \mathrm{c}_{2}, \mathrm{c}$ and $\mathrm{c}_{0}$ respectively, including handling costs. Only when the transportation cost of $\mathrm{P}_{1}$ to $\mathrm{P}_{2}$ meets formula (2) and the time meets the requirements of the supply chain, can the mode of sea transportation be selected. Because shipping cargo is not sensitive to time, time is not considered here.

$$
L_{e} c_{1}+L c+L_{e}^{\prime} c_{2} \leq L_{0} c_{0}
$$

Suppose the pricing policy of sea transportation is average total cost + profit $^{[6]}, \mathrm{C}_{\mathrm{s}}$ is the total cost of shipping and $\mathrm{r}$ is the profit per unit volume, then

$$
c=\frac{C_{s}}{Q_{s}}+r
$$

Substitute it into equation (2). $\mathrm{P}_{1}$ is a boundary point. Take the equal sign of formula (2), 


$$
\begin{gathered}
L_{e}=\frac{L_{0} c_{0}-L_{e}^{\prime} c_{2}-L\left(\frac{C_{s}}{Q_{s}}+r\right)}{c_{1}} \\
\frac{d L_{e}}{d Q_{s}}=\frac{L C_{s}}{c_{1} Q_{s}^{2}}
\end{gathered}
$$

From equations (4) and (5), $\mathrm{L}_{\mathrm{e}}$ and $\mathrm{Q}_{\mathrm{s}}$ change in the same direction, that is, the increase in the transport volume of the shipping passage will increase the continental depth of the economic belt, but the increase rate of Le gradually decreases with the increase of $\mathrm{Q}_{s}$. In other words, the effect of the transportation volume of the shipping passage on the expansion of the economic belt is slowing down, and the growth of the scope of the economic belt is limited, and it can not expand indefinitely. $c_{1}$ and $c_{2}$ have negative effects on $L_{e}$, indicating that improving the capacity and smoothness of port collection and distribution will help to expand the scope of economic belt. The reverse effect between $L_{e}$ and $\mathrm{L}_{\mathrm{e}}$ ' indicates that there is a trade-off among the landward depth of different regions of the economic belt when other conditions are constant. If $r$ is high, $L_{e}$ will be small, so the attitude of the shipping company to the profit level will affect the scope of the economic belt. If the economic benefits are more important, the economic belt will be narrow, which will promote the concentration of seaworthy industries near the port, but may lose the longdistance inland goods with more alternative transport mode.

\subsection{The Effect Coefficient $\sigma_{\mathrm{s}}$}

The required $\sigma_{\mathrm{s}}$ is $\mathrm{dQ}_{\mathrm{s}} / \mathrm{dL}_{\mathrm{e}}$, that is, the change rate of $\mathrm{Q}_{\mathrm{s}}$ to $\mathrm{L}_{\mathrm{e}}$. However, this is not a mathematical problem. It is necessary to analyze the working principle displayed from the causal relationship to obtain it. It cannot be simply treated with the reciprocal of the above results. The increase of the landward depth of the economic belt makes the transportation strategy of the shipping passage suitable for further industrial trade, and $\mathrm{Q}_{\mathrm{s}}$ will inevitably increase. However, the density of industrial distribution or the distribution of transportation demand is generally not uniform in space. In this paper, the form of $\sigma_{\mathrm{s}}$ is studied based on the plume model currently applied in the research of port hinterland. The plume model was originally used to calculate, predict and describe the spatial pollutant concentration in the downwind direction of the pollution source, and here it is used to describe the spatial distribution of transport demand "concentration" centered on the shipping passage and its port, so as to determine the increase of $Q_{s}$ with the increase of $L_{e}$.

Let $\beta_{1}$ be land transport demand concentration of $\mathrm{H}_{1}$, that is, the simplified plume model, see equation (6). $\alpha_{1}$ is the conditional coefficient of $\mathrm{H}_{1}$, which is generally related to the basic conditions and service level of the port. $\theta_{1}$ is the intensity of $H_{1}$, which is expressed by the port transport volume. $\mu_{1}$ is the traffic condition coefficient between $\mathrm{H}_{1}$ and inland area. $\mathrm{R}_{1}$ is the correlation degree between $\mathrm{H}_{1}$ and demand point $\mathrm{P}$, as shown in formula (7), where $\mathrm{n}$ and $\mathrm{v}$ are the permanent urban population and industrial output value of $\mathrm{H}_{1}$ and $\mathrm{P}$ respectively ( $\mathrm{Li}$ zhenfu, 2015, see reference [7]).

$$
\begin{aligned}
& \beta_{1}=\frac{\alpha_{1} \theta_{1} \mu_{1} R_{1}}{4 \pi L_{e}^{2}} \\
& R_{1}=\frac{\sqrt{n_{1} v_{1} n_{P} v_{P}}}{L_{e}^{2}}
\end{aligned}
$$

$\beta_{1}$ varies with $L_{e}$, so the transport volume $Q_{1}$ in a certain area around the port is the integral of $\beta_{1}$ on the area $\mathrm{S}_{\mathrm{H} 1}$, which can be converted into the integral on the landward depth $\mathrm{L}_{e}$, the economic belt area at the end of $\mathrm{H}_{1}$ can be approximately regarded as a $1 / 4$ circle, as shown in figure 1, get formula (8). That of end $\mathrm{H}_{2}$ is in the same way.

$$
\begin{aligned}
& Q_{1}=\int_{0}^{\frac{\pi L_{e}^{2}}{4}} \frac{\alpha_{1} \theta_{1} \mu_{1} R_{1}}{4 \pi L_{e}^{2}} d S_{H 1}=\int_{0}^{L_{e}} \frac{\alpha_{1} \theta_{1} \mu_{1} R_{1}}{4 \pi L_{e}^{2}} \cdot \frac{\pi}{2} L_{e} d L_{e} \\
& \text {................. radius from terminal port to boundary of the economic belt } \\
& \text { - - - - boundary after expansion of the economic } \\
& \text { boundary before expansion of the economic belt } \\
& -\cdots-\text { coastline }
\end{aligned}
$$

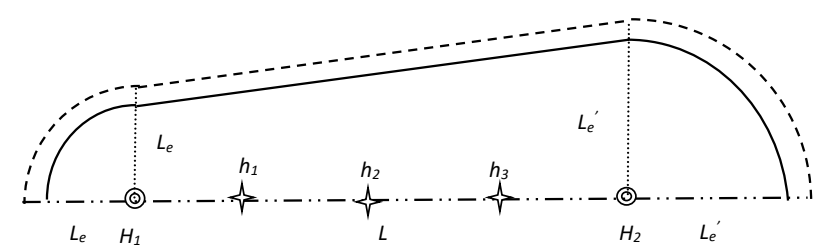

Figure.1 Geometric diagram for calculating transport demand in maritime transportation economic belt

$\mathrm{L}_{\mathrm{e}}$ and $\mathrm{L}_{\mathrm{e}}$ ' are usually not equal, and the border and coastline of the economic belt are not straight. As a preliminary study of this kind of problem, the area enclosed by two $1 / 4$ circles and a right-angled trapezoid is used here to indicate the approximate scope of the maritime transportation economic belt, as shown in Figure 1. The transport demand within the two $1 / 4$ circles is represented by the demand concentration plume model of $\mathrm{H}_{1}$ and $\mathrm{H}_{2}$ respectively, and the transport demand of the trapezoid area is represented by the demand concentration plume model generated by the shipping passage L. In order to obtain the change rate of $\mathrm{Q}_{\mathrm{s}}$ to $\mathrm{L}_{\mathrm{e}}, \mathrm{L}_{\mathrm{e}}$ ' needs to be represented by $L_{e}$. Let the initial state $L_{e}{ }^{\prime}(0)=\lambda L_{e}(0)$, the landward depth of the economic belt increase $\Delta$ means both $\mathrm{L}_{\mathrm{e}}(0)$ and $\mathrm{L}_{\mathrm{e}}{ }^{\prime}(0)$ increase $\Delta$, then $\mathrm{L}_{\mathrm{e}}{ }^{\prime} / \mathrm{L}_{\mathrm{e}}$ becomes $\left(\lambda \mathrm{L}_{\mathrm{e}}(0)+\Delta\right) /\left(\mathrm{L}_{\mathrm{e}}(0)+\Delta\right) . \Delta$ is small enough compared to $\mathrm{L}_{\mathrm{e}}(0)$, so $\mathrm{L}_{\mathrm{e}}$ ' can still be approximated to $\lambda \mathrm{L}_{\mathrm{e}}$ in the calculation. So $\mathrm{Q}_{2}$ at the end of $\mathrm{H}_{2}$ is

$Q_{2}=\int_{0}^{L_{e}^{\prime}} \frac{\alpha_{2} \theta_{2} \mu_{2} R_{2}}{4 \pi L_{e}^{\prime 2}} \cdot \frac{\pi}{2} L_{e}^{\prime} d L_{e}^{\prime}=\int_{0}^{L_{e}} \frac{\alpha_{2} \theta_{2} \mu_{2} R_{2}}{4 \pi\left(\lambda L_{e}\right)^{2}} \cdot \frac{\pi}{2} \lambda L_{e} \lambda d L_{e}$

There may be a number of other ports on $\mathrm{L}$, and the distribution of actual transport demand within the belt varies widely. In order to have a general theory, the distribution of transport demand in the trapezoid region is represented by the demand concentration generated by the weighted average state of ports on L, and it diffuses inland from the coastline with the same change of 
concentration. The transport volume $\mathrm{Q}_{\mathrm{T}}$ in the trapezoid region is the integral of the demand concentration $\beta_{\mathrm{T}}$ on the area $S_{T}$, which can also be converted to the integral on $\mathrm{L}_{\mathrm{e}}$, as shown in equation (10). $\mathrm{m}$ is the number of ports on $\mathrm{L}, \gamma_{i}{ }^{(\alpha)}, \gamma_{i}{ }^{(\theta)}, \gamma_{i}{ }^{(\mu)}, \gamma_{i}{ }^{(R)}$ are the weights of each port on $\alpha, \theta$, $\mu$ and $\mathrm{R}$ respectively.

$$
\begin{gathered}
Q_{T}=\int_{0}^{\frac{\left(L_{e}+L_{e}^{\prime}\right) L}{2}} \beta_{T} d S_{T} \\
=\int_{0}^{\frac{(\lambda+1) L_{e} L}{2}} \frac{\sum_{i=1}^{m} \gamma_{i}^{(\alpha)} \alpha_{i} \sum_{i=1}^{m} \gamma_{i}^{(\theta)} \theta_{i} \sum_{i=1}^{m} \gamma_{i}^{(\mu)} \mu_{i} \sum_{i=1}^{m} \gamma_{i}^{(R)} R_{i}}{4 \pi L_{e}^{2}} d S_{T} \\
=\int_{0}^{L_{e}} \frac{\sum_{i=1}^{n} \gamma_{i}^{(\alpha)} \alpha_{i} \sum_{i=1}^{n} \gamma_{i}^{(\theta)} \theta_{i} \sum_{i=1}^{n} \gamma_{i}^{(\mu)} \mu_{i} \sum_{i=1}^{n} \gamma_{i}^{(R)} R_{i}}{4 \pi L_{e}^{2}} \cdot \frac{(\lambda+1) L}{2} d L_{e}
\end{gathered}
$$

Let $\alpha_{i} \theta_{i} \mu_{i} R_{i}=f_{i}, \quad F$ is the product of the weighted average value of the port parameters in $\mathrm{L}$, then $\sigma_{\mathrm{s}}$ is

$$
\sigma_{s}=\frac{d\left(Q_{1}+Q_{2}+Q_{T}\right)}{d L_{e}}=\frac{f_{1}+f_{2}}{8 L_{e}}+\frac{F(\lambda+1) L}{8 \pi L_{e}^{2}}
$$

It can be seen that the increase extent of $\mathrm{Q}_{\mathrm{s}}$ also decreases with the increase of $L_{e}$, and the effect of the expansion of the economic belt on the growth of the transport volume of the shipping passage is also slowing down. The comprehensive capacity of ports, the length of the shipping passage and the difference in the landward depth of the economic belt all have positive effects on $\sigma_{\mathrm{s}}$.

Substituting equations (5) and (11) into (1), the form of the symbiotic equation with the transport volume of the shipping passage and the landward depth of its economic belt as the scale variables is as follows:

$$
\left\{\begin{array}{c}
\frac{d L_{e}}{d t}=a_{e} L_{e}\left[-1-\frac{L_{e}}{K_{e}}+\frac{L C_{s}}{c_{1} Q_{s}^{2}} \cdot \frac{Q_{s}}{K_{s}}\right] \\
\frac{d Q_{s}}{d t}=a_{s} Q_{s}\left[1-\frac{Q_{s}}{K_{s}}+\left(\frac{f_{1}+f_{2}}{8 L_{e}}+\frac{F(\lambda+1) L}{8 \pi L_{e}^{2}}\right) \frac{L_{e}}{K_{e}}\right]
\end{array}\right.
$$

It can be seen that the interaction coefficients $\sigma_{\mathrm{e}}, \sigma_{\mathrm{s}}$ of shipping passage and its economic belt vary with the scale variables, and are not constant coefficients like the general logistic equations. It shows that the interaction force is related to the value of the scale variables, which is not constant in the evolution process of the marine transportation economic belt, and decreases with the increase of the scale variables. It shows that the mutual promotion of the two subsystems in the early stage of the development of the maritime transportation economic belt is relatively large when other factors remain unchanged, and the mutual promotion of the two subsystems is not obvious after the mature stage of the development with a relatively large scale, at this time, the effect of investment and construction is not as good as the initial stage.

\section{CONCLUSION}

There is a symbiotic relationship between the shipping passage and its coastal economic belt, which together constitute the maritime transportation economic belt. Its evolution process can be studied by using logistic equation, in which the independent natural growth coefficient of economic belt is negative. When the scale variables are the transport volume of the shipping passage and the landward depth of its economic belt, the interaction coefficient can be obtained by using the plume model to express the demand concentration in the geometric geographic range and cost comparison, showing a way to quantify the interaction relationship and realizing the practicability of its logistic equation. The study found that the two scale variables are increasing functions of each other, but the interaction gradually slows down with the increase of their scale. Improving the capacity and smoothness of cargo collection and distribution through port will help to expand the scope of the economic belt. The increase of profit level of shipping companies will reduce the landward depth of economic belt. Longer shipping passage, higher total shipping costs, and lower land transport cost will make the impact coefficient of shipping volume larger. A higher comprehensive port capacity, a longer shipping passage and a larger difference in the landward depth of the economic belt will make the influence coefficient of the landward depth of the economic belt larger.

\section{ACKNOWLEDGMENT}

This work was supported by the Natural Science Foundation Project of Fujian Province under Grant [number 2019J01687]; Science and Technology Project of Fujian Education Department under Grant [number JT180258, JT180257].

\section{REFERENCES}

1. Z. Wenchang, J. Fengjun, and F. Jie, Traffic Economic Belt. Beijing: Science Press, 2002, pp.27-42.

2. T. Tsekeris, "Domestic transport effects on regional export trade in Greece", Research in Transportation Economics, vol. 61, pp. 2-14, March 2017.

3. C. Clott, B. C. Hartman, "Supply chain integration, landside operations and port accessibility in metropolitan Chicago", Journal of Transport Geography, vol.51, pp. 130-139, February 2016.

4. W. Bart, J. Milan, "Analysis, modeling, and assessing performances of supply chains served by longdistance freight transport corridors", Journal of Sustainable Transportation, vol. 13, pp. 278-293, April 2019.

5. Y. Dong, J. Liping, K. Y. Ng. Adolf, "One Belt one Road, but several routes: A case study of new emerging trade corridors connecting the Far East to Europe", Transportation Research Part A: Policy and Practice, vol. 117, pp. 190-204, November 2018. 
6. Y. Jin, International Shipping Economics. Beijing: China Communications Press, 2014, pp. 109-112.

7. L. Zhenfu, T. Xiaowen, "Hinterland Plume Model Research on the Evolution of Port Hinterland Under Big Bohai Circle”, vol. 35, pp. 116-123, June 2015. 